\title{
Isolated Fetal Corpus Callosum Agenesis: Diagnosis and Management Difficulties
}

\author{
Achour Radhouane $^{1^{*}}$, Ksibi Imen ${ }^{2}$, Ben Jamaa Nadia ${ }^{3}$, Aloui Nadia ${ }^{4}$, Kacem Samia ${ }^{1}$ and Neji Khaled ${ }^{1}$ \\ ${ }^{1}$ Department of Emergency, Center of Maternity and Neonatology of Tunis, Faculty of Medicine of Tunis, El Manar University Tunisia \\ ${ }^{2}$ Neonatal Intensive Care Unit, Center of Maternity and Neonatology of Tunis, University Tunis El Manar, Tunisia \\ ${ }^{3}$ Department of Fetopathology, Center of Maternity and Neonatology of Tunis, Faculty of Medicine of Tunis, El Manar University Tunisia \\ ${ }^{4}$ Department of Radiology, Center of Maternity and Neonatology of Tunis, University Tunis El Manar, Tunisia \\ "Corresponding author: Achour Radhouane, Maternity and Neonatalogy center tunis, Tunisia, Tel: + 21698549398; E-mail: radhouane.a@live.com
}

Received date: September 21, 2016; Accepted date: October 12, 2016; Published date: October 20, 2016

Copyright: (c) 2016 Radhouane A, et al. This is an open-access article distributed under the terms of the Creative Commons Attribution License, which permits unrestricted use, distribution, and reproduction in any medium, provided the original author and source are credited.

\begin{abstract}
The combined prevalence of partial or complete agenesis of the corpus callosum (ACC) is estimated at $0.02-0.5 \%$. This common brain malformation mainly due to a prenatal abnormality commissuration. We report a case of diagnosis of isolated fetal CCA. Magnetic resonance imaging appears to be an important adjunct as it allows direct visualization. The prognosis in isolated agencies of the corpus callosum remains uncertain.
\end{abstract}

Keywords: Corpus callosum; Agenesis; Prenatal; Prognosis

\section{Introduction}

The corpus callosum is the largest commissural structure in the human brain, formed by more than 190 million cross-hemispheric axons that are known to exhibit excitatory function [1]. The combined prevalence of partial or complete agenesis of the corpus callosum (ACC) is estimated at $0.02-0.5 \%$ [2]. This common brain malformation mainly due to a prenatal abnormality commissuration [1].

The corpus callosum (CC) can be assessed on ultrasound by direct visualization, or by finding of indirect signs suggestive of corpus callosum agenesis, such as moderate distension of the occipital ventricle and the ventricular communications, absence of the cavum septi pellucidi, are often the reason for detection in a screening population.

Magnetic resonance imaging appears to be an important adjunct as it allows direct visualization. It can confirm ACC, assess whether this is complete or partial and detect coexisting brain abnormalities.

The prognosis in isolated agencies of the corpus callosum remains uncertain, but neurodevelopmental outcome in this case was reported in the literature and suggested normal outcome in about $65-75 \%$ of cases.

Each case should be discussed during the pluridisciplinary prenatal diagnostic discussion and termination of pregnancy is often advised in these circumstances.

\section{Case Report}

A 33 year old woman, gravida 1, para 1, non-consanguineous marriage, was referred from a local clinic with suspected fetal agenesis of the corpus callosum at 26 weeks gestation.

After a reference ultrasound examination, we released a fetal MRI which confirmed the diagnosis of agenesis of the corpus callosum without any associated abnormalities (Figures 1 and 2).

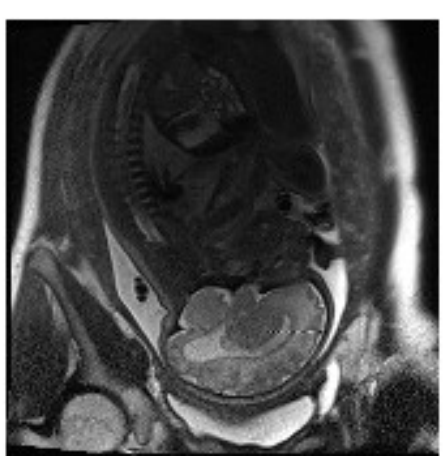

Figure 1: Fetal MRI. Corpus callosum agenesis.

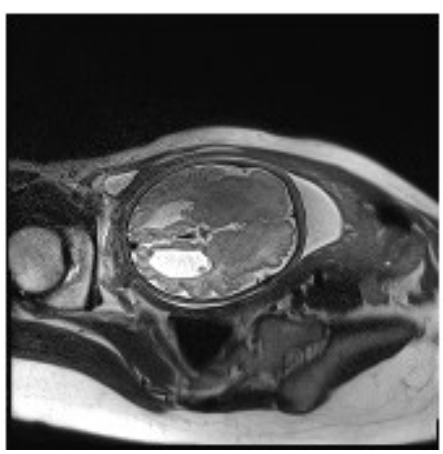

Figure 2: Fetal MRI. Corpus callosum agenesis.

Monitoring of the pregnancy was uneventful with normal fetal development. 
Page 2 of 2

An elective caesarean delivery was performed at 38 weeks, resulting in a healthy male infant weighing $3400 \mathrm{~g}$. He has 2 years old age, with normal psychomotor development.

\section{Conclusion}

Agenesis of the corpus callosum (ACC) can result from genetic, infectious, vascular or toxic causes, and it can be either complete (CACC) or partial (PACC) [3-5].

It may be an isolated anomaly or an associated anomaly as part of various syndromes.

Long ago; before the era of prenatal diagnosis, the CCA, is diagnostic in post natal in children with neurological symptoms $[5,6]$.

PACC can be diagnosed reliably and characterized in prenatal life since the late 1980s and that awareness of typical findings should permit more frequent detection of this anomaly in utero [7].

Prenatal MRI is a valuable complementary technique for either diagnosis of corpus callosum agenesis and depiction of associated neurologic abnormalities [8].

Because prognosis depends on associated anomalies, a detailed examination of fetal intracranial anatomy should be performed.

A multidisciplinary team consisting of experienced clinicians should be involved to help parents make informed decisions [9].

\section{References}

1. Jakab A, Kasprian G, Schwartz E, Gruber GM, Mitter C, et al. (2015) Disrupted developmental organization of the structural connectome in fetuses with corpus callosum agenesis. NeuroImage 111: 277-288.
2. Aicardi J, Chevrie JJ, Baraton J (1987) Agenesis of corpus callosum. In: Vinken PJ, Bruyen GW, Klawans HL editors, Handbook of clinical neurology. Revised series. Brain malformations. Amsterdam, North Holland.

3. Atlas SW, Zimmerman RA, Bilaniuk LT (1986) Corpus callosum and limbic system: Neuroanatomic MR evaluation of developmental anomalies. Radiology 160: 355-362.

4. Jones K, Smith DW, Ulleland C, Stressguth AP (1973) Pattern of malformations in offspring of chronic alcoholic mothers. Lancet 1 : 1267-1271.

5. Sotiriadis A, Makrydimas G (2012) Neurodevelopment after prenatal diagnosis of isolated agenesis of the corpus callosum: an integrative review. Am J Obstet Gynecol 206: e1-5.

6. Serur D, Jeret JS, Wisniewski K (1988) Agenesis of the corpus callosum: Clinical, neuro radiological and cytogenetic studies. Neuropediatrics 19: 87-91.

7. Blum A, Andre M, Broulle P, Husson S, Leheup B (1990) Prenatal echographic diagnosis of corpus callosum agenesis: The Nancy experience 1982-1989. Genetic Counseling 38: 115-126.

8. Achour Radhouane, Neji Khaled (2016) Corpus callosum agenesis: Role of fetal magnetic resonance imaging. Asian Pacific Journal of Reproduction 5: 263-265.

9. Vasudevan C, McKechnie L, Levene M (2012) Long-term outcome of antenatally diagnosed agenesis of corpus callosum and cerebellar malformations. Seminars in Fetal \& Neonatal Medicine 17: 295-300. 